\title{
Molecular and Phylogenetic Characterization of Cytokine Genes from Seba's Short-Tailed Bat (Carollia perspicillata)
}

\author{
Ann C. Cogswell-Hawkinson ${ }^{1}$, Mitchell E. McGlaughlin ${ }^{1}$, Charles H. Calisher ${ }^{2}$, Rick Adams ${ }^{1}$ and \\ Tony Schountz ${ }^{*}, 1$ \\ ${ }^{1}$ School of Biological Sciences, University of Northern Colorado, $50120^{\text {th }}$ Street, Greeley, CO 80369, USA \\ ${ }^{2}$ Arthropod-Borne and Infectious Diseases Laboratory, Department of Microbiology, Immunology and Pathology, \\ College of Veterinary Medicine and Biomedical Sciences, Colorado State University, Fort Collins, CO 80523, USA
}

\begin{abstract}
Bats (order Chiroptera) represent nearly one fourth of the approximately 5,000 known species of mammals, yet many aspects of their biology are poorly understood. Bats of certain species are well known reservoirs of rabies virus but within the past few years bats also have been identified as reservoirs or potential reservoirs of several other important human and livestock pathogens. In some instances, these viruses may cause persistent infections without recognized pathology in the bat host. Even though these are medically important viruses, little is known as to how bat immune systems engage viruses or how these viruses may evade a sterilizing host immune response. We have recently initiated research on Seba's short-tailed fruit bat (Carollia perspicillata), which may be a reservoir for a newly discovered coronavirus. We cloned and characterized cDNAs of four cytokine genes; tumor necrosis factor, interleukin10, interleukin-23a, and granulocyte macrophage stimulating factor. Our sequence analysis shows these genes are highly conserved with regard to orthologous sequences and they provide some value for resolving phylogenetic relationships between mammals. This work represents a first step in developing an infection and immunology model for a New World coronavirus in bats.
\end{abstract}

Keywords: Chiroptera, Cytokines, Carollia perspicillata, Seba's short-tailed fruit bat.

\section{INTRODUCTION}

In recent years, several zoonotic viral disease agents have been isolated from or detected in bats [1]. For some of these viruses bats are known reservoir hosts, and for others they are suspected reservoirs or incidental hosts. Hendra virus, hosted by pteropid bats [2], was first identified from cases of fatal equine and human respiratory disease in Australia [3]. Nipah virus, also hosted by pteropid bats [4], has been associated with multiple outbreaks of human disease in Cambodia, Malaysia, India and Bangladesh, with casefatality rates as high as 70\% [5]. Lyssaviruses, including rabies virus, have been detected in bats throughout the world, except in Antarctica, which has no bats [6]. Severe acute respiratory syndrome coronavirus (SARS-CoV)-like viruses have been detected in Chinese horseshoe bats [7], and it is thought SARS-CoV is a direct descendent of these bat-borne viruses. Recent serological and molecular evidence suggests that ebolaviruses and Marburg virus are hosted by fruit bats in Africa [8]. While some infected reservoir host bats may show pathology, others appear to be asymptomatic [9]. Currently, more than 100 viruses have been identified from or detected in bat tissues, suggesting that bats are significantly underappreciated as hosts and vectors for human pathogens. Studies evaluating immune competency of bats are only now beginning to be conducted [10-15] and

*Address correspondence to this author at the School of Biological Sciences, University of Northern Colorado, 501 20th Street, Greeley, CO 80369, USA; Tel: 01970351 2923; Fax: 019703512335 ;

E-mail: tony.schountz@unco.edu the emergence of cost-effective transcriptome sequencing should permit rapid advances in developing tools for assessing bat immune responses.

Three viruses have been detected in Seba's short-tailed bats (Carollia perspicillata); an unspecified betaretrovirus, Venezuelan equine encephalitis virus (VEEV) and a group 1 coronavirus [16-18]. It is unknown whether bats of this species are reservoirs for these viruses or whether infections were incidental. Because these bats are found from central Mexico to southern Brazil [19], it is important to determine their possible role in maintenance and dissemination of these and other viruses. For this reason, we have begun a project to determine susceptibility of Seba's bat to one of these viruses with the intent of studying infection of these bats with a novel coronavirus discovered in Trinidad and Tobago [18]. While many novel coronaviruses have been recently discovered in bats, no infection models have been established.

Since the role of the host response is important in resistance and susceptibility, it will be necessary to develop methods for evaluating immune responses in the bats. It is likely that some bats may become persistently-infected without disease and that the viruses have evolved immune evasion strategies. We have successfully cloned partial sequences of four cytokine genes from Seba's short-tailed bat: tumor necrosis factor (TNF), interleukin-10 (IL-10), IL23a, and granulocyte-macrophage colony stimulating factor (GM-CSF). We present here molecular and phylogenetic analyses of the sequences. 


\section{MATERIALS AND METHODS}

\section{Bats}

The University of Northern Colorado (UNC) bat colony comprises neotropical fruit bats of two species, $C$. perspicillata and Artibeus jamaicensis (Jamaican fruit bat). These are maintained together in a $36 \mathrm{~m}^{3}$ room that allows unrestrained free-flight of all individuals. The colony has been closed for 16 years and is rabies virus-free; PCR screening of rectal swab samples from 20 of these bats has failed to detect coronaviruses (K. Holmes, pers. comm.). The bats have access to roosting areas in the form of ceiling-hung baskets and various cloth drapes and are maintained under a light-cycle of 12L:12D. Ambient temperature is maintained between 20 and $25^{\circ} \mathrm{C}$ and humidity between $50 \%$ and $70 \%$ by a computer-controlled HVAC system. Bats are fed daily at midday with grueled apples, monkey chow (Harlan Teklad, Denver, CO), molasses, nonfat dry milk, cherry gelatin with raisins, and fresh bananas. In addition, a variety of other fruits, including mango, papaya, cantaloupe, banana, grapes, and watermelon, are provided in food trays and also arrayed on skewers throughout the room to stimulate foraging behavior. For additional enrichment, artificial trees and vines are provided.

All procedures for the present work were approved by the University of Northern Colorado Institutional Animal Care and Use Committee and were in compliance with the USA Animal Welfare Act. Euthanasia was performed by respiratory hyperanesthesia with isoflurane followed by thoracotomy.

\section{RNA Extraction}

A single cell suspension of splenocytes was prepared by gentle disruption of Seba fruit bat spleens between the ends of sterile frosted glass microscope slides. Red blood cells were lysed with AKC Lysis Buffer (Cambrex Biosciences, East Rutherford, $\mathrm{NJ}$ ) for $5 \mathrm{~min}$ at room temperature followed by $3 \mathrm{x}$ wash in PBS ( $\mathrm{pH}$ 7.4). Splenocytes were cultured in $5 \%$ FBS RPMI-1640 and activated in $2 \mu \mathrm{g} / \mathrm{ml}$ concanavalin A and $2 \mu \mathrm{g} / \mathrm{ml}$ E. coli LPS (Sigma, St. Louis, MO) overnight at $37^{\circ} \mathrm{C}$ under $7 \% \mathrm{CO}_{2}$. RNA was extracted according to manufacturer's instructions using the Serious RNA Purification ${ }^{\mathrm{TM}}$ RNA Cell kit (Gentra Systems, Minneapolis, $\mathrm{MN}$ ) and aliquots were stored at $-70^{\circ} \mathrm{C}$ until used.

\section{Reverse Transcription-Polymerase Chain Reaction}

RNA was reverse-transcribed using iScript ${ }^{\mathrm{TM}}$ cDNA synthesis kit (BioRad, Hercules, CA, USA) using a mixture of random hexamers and oligo-dT primers. PCR was performed using degenerate primers (Table 1) with the PCR Core kit (Qiagen, Valencia, CA). Amplification was initially performed using 35 cycles of $95^{\circ} \mathrm{C}$ for 30 seconds, annealing at $58^{\circ} \mathrm{C}$ for 30 seconds, and extension at $72^{\circ} \mathrm{C}$ for 1 minute. After 35 cycles, samples were incubated at $72^{\circ} \mathrm{C}$ for 10 minutes and then held at $4^{\circ} \mathrm{C}$. For genes that failed to amplify with $58^{\circ} \mathrm{C}$ annealing, the annealing temperature was lowered to $50^{\circ} \mathrm{C}$ for the first five cycles, then at $58^{\circ} \mathrm{C}$ for 30 cycles. PCR products were then resolved on a $1 \%$ agarose gel to verify amplification.

\section{Cloning of PCR Products}

Amplicons were cloned into TOPO-TA Sequencing (Invitrogen, Carlsbad, CA) vector according to the manufacturer's directions. Vectors were then used to transform competent DH5 $\alpha$ E. coli cells that were then plated on LB/Amp. Several colonies were chosen from each gene and screened by PCR to verify the correct plasmid insert. Colony screening was done by PCR (PCR Master Mix, Madison, WI) with primers used for the original amplifications. Selected colonies were then incubated overnight at $37^{\circ} \mathrm{C}$ in $4 \mathrm{~mL}$ of LB broth containing $50 \mu \mathrm{g} / \mathrm{ml}$ ampicillin. Plasmids were purified using a Qiagen QIAprep spin Miniprep kit (Valencia, CA), and recovery was verified using a $1 \%$ agarose gel.

\section{Rapid Amplification of cDNA Ends (RACE)}

RACE PCR was used to obtain additional 5' and 3' sequences of Seba's short-tailed bat IL-23a, IL-10, and GMCSF genes. 5' regions were extended for all three genes, but 3' RACE fragments were not obtained. RACE was performed using a SMART ${ }^{\mathrm{TM}}$ RACE cDNA Amplification kit (Clontech, Mountain View, CA). Primers for RACE PCR were designed from conserved regions in previously obtained sequences described in section 2.4. RACE primers are included in Table $\mathbf{2}$.

\section{Sequence Analysis}

Sequencing reactions were performed using $\mathrm{T} 7$ and $\mathrm{T} 3$ primers with Big Dye Terminator (Applied Biosystems,

Table 1. Primer Sequences Used to Clone Partial Cytokine cDNAs from Seba's Short-Tailed Bat

\begin{tabular}{|c|l|l|c|}
\hline Gene & \multicolumn{1}{|c|}{ Forward } & Reverse & Size (bp) \\
\hline \hline TNF & GCGACGTAGAACTGGCAGAGG & CTGCGAAGTCTAGGTATTTGG & 648 \\
\hline IL-10 & CTCAGCACTGCTCTGTTGCC & $\begin{array}{l}\text { 1 GATGTCAAACTCAGTCATGG } \\
2 \text { GATGTCAAACTCATTCATGG }\end{array}$ & CTGCTCCGTGGGCAAAGACC \\
\hline IL-23 & 1 AGCCAGATCTGAGAAGAAGG & & 311 \\
& 2 AGCCAGATCTGAGAAGCAGG & 1 ATGGTCAAGGGGCCCTTGAG & 2 ATGGTCAAGGCGCCCTTGAG \\
& 1 CTGGAGGATGTGGCTGCAG & & \\
& 2 CTGGAGGATGTGGCTGAAG & & \\
\hline
\end{tabular}


Table 2. RACE Primer Sequences Used to Amplify 5' cDNA Regions of Seba's Bat Cytokines

\begin{tabular}{|c|l|l|}
\hline Gene & \multicolumn{1}{|c|}{ Forward } & Reverse \\
\hline \hline IL-10 & TCGCCCTTCTCAGCACTGCTCTGTTGCC & GTTTGGGCAGGTTGGCTGGGAAGTCACTG \\
\hline IL-23 & TGCCTGAAGGCAGCGGCCCCTCTTG & TCCACACGTCCATGGCTGGATGTGC \\
\hline GM-CSF & CCTGCTTCTTCTGGGCACAGTGGCTTGC & GCGCCCTTGAGTATGGTGAGGTTGCCCC \\
\hline
\end{tabular}

Foster City, CA). Sequences were edited and contigs built using Sequencher (GeneCodes, Ann Arbor, MI) and BLAST was used to identify genes. Amino acid alignments were made using orthologous cytokine sequences from human (Homo sapiens), chimpanzee (Pan troglodytes), rhesus monkey (Macaca mulatta), domestic dog (Canis lupus familiaris), domestic cat (Felis catus), and house mouse (Mus musculus). Accession numbers are listed for each protein in Table 3. The CLUSTAL algorithm in MacVector sequence analysis software (Cary, NC) was used to perform the alignments. Sequences were deposited into GenBank (TNF, EF653909; GM-CSF, EF653911; IL-23a, EU223817; IL-10, EF653910).

\section{Phylogenetic Analysis}

Phylogenetic analyses were conducted using amino acid sequences collected from Seba's short-tailed bat and orthologous sequences obtained from GenBank. Orthologous sequences were obtained from a single representative of as many taxonomic groups as possible. The following orthologous sequences were used (see Table $\mathbf{3}$ for GenBank accession numbers); TNF from human, chimpanzee, rhesus monkey, domestic dog, domestic cat, house mouse, horse (Equus caballus), cattle (Bos taurus), pig (Sus scrofa), and chicken (Gallus gallus); IL-10 from human, chimpanzee, rhesus monkey, domestic dog, domestic cat, house mouse, horse, cattle, pig, and chicken; IL-23a from human, chimpanzee, rhesus monkey, domestic dog, domestic cat, house mouse, horse, cattle, pig, and platypus (Ornithorhynchus anatinus); and GM-CSF from human, chimpanzee, rhesus monkey, domestic dog, domestic cat, house mouse, horse, cattle, pig, and chicken.

All sequences were aligned using the CLUSTAL algorithm in MacVector sequence analysis software. PAUP* $4.0 \mathrm{~b} 10$ [20] was used to perform maximum parsimony analyses for each region. All characters were weighted equally and the exhaustive search procedure was used to determine the most parsimonious tree. Trees were rooted using chicken as an outgroup for GM-CSF, TNF, and IL-10, and platypus for IL-23a. Support for branching patterns were analyzed using a parsimony bootstrap procedure with 1000 replicates.

\section{RESULTS}

\section{Strategy for Cloning Bat Cytokine Genes}

We adapted a similar degenerate PCR cloning strategy previously used by us with wild rodents [21, 22]. Orthologous sequences of several mammalian cytokine genes were aligned and degenerate primer sets were designed (Table 1). Total RNA was extracted from concanavalin A and LPS-stimulated splenocytes to enhance transcription of cytokine genes. RT-PCR was then performed and amplicons were cloned and sequenced. We aligned translated bat and orthologous sequences to identify conserved amino acid regions and analyze phylogenetic relationships.

\section{TNF}

The cloned cDNA of Seba's short-tailed bat TNF was $675 \mathrm{nt}$ and encodes approximately $96 \%$ of the TNF coding region. The nucleotide sequence was translated using MacVector's default translation table and then aligned with orthologous mammalian sequences. The nucleotide sequence encodes nearly all of the TNF polypeptide (226 residues) and exhibits $82 \%$ identity and $84 \%$ similarity to human TNF (Fig. 1), and $79 \%$ identity and $86 \%$ similarity to Leschenault's Rousette bat TNF [15]. The gene is highly conserved between species and shares a nearly identical transmembrane domain with orthologous TNF (residues 3258) [23]. Serine residues at positions 83 and 84 , the putative cleavage point for the release of soluble TNF homotrimer, appear to be similar to those of human, chimpanzee, rhesus monkey, dog, cat, and mouse TNF. The cysteine residues at positions 148 and 180 for intrachain disulfide bond formation are conserved in this bat [24]. A conserved histidine is present in the bat sequence at position 194, and is necessary for biological activity of TNF [25]. Residues L115, S165, and E225 are involved in receptor interaction of

Table 3. Polypeptide Accession Numbers of Sequences Used from the National Center for Biotechnology Information

\begin{tabular}{|c|c|c|c|c|}
\hline Protein: & TNF & GM-CSF & IL-10 & IL-23 \\
\hline \hline Human & NP_000585.2 & AAU21240.1 & CAG46825 & AAH66267.1 \\
\hline Chimpanzee & NP_001038976.1 & XP_527005.2 & XP_525040.1 & XP_522436.1 \\
\hline Rhesus monkey & NP_001040614.1 & NP_001028121.1 & ABI63892.1 & XP_538231.2 \\
\hline Dog & NP_001003244.4 & NP_001003245.1 & NP_001003077.1 & ABB01676.1 \\
\hline Cat & NP_001009835.1 & AAN11308.1 & AAC64708.1 & NP_112542.1 \\
\hline House mouse & NP_038721.1 & NP_034099.2 & NP_034678.1 & \\
\hline
\end{tabular}



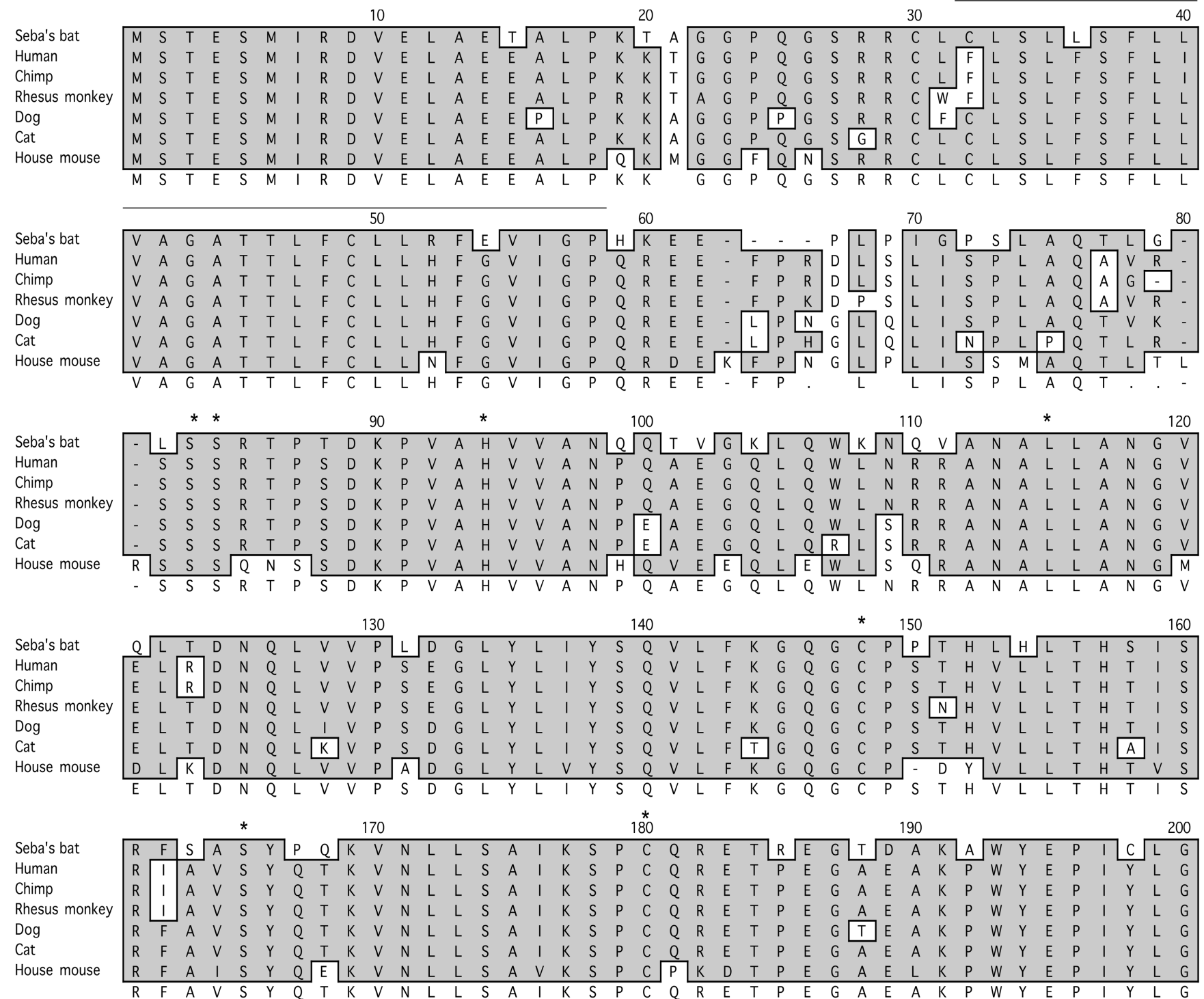

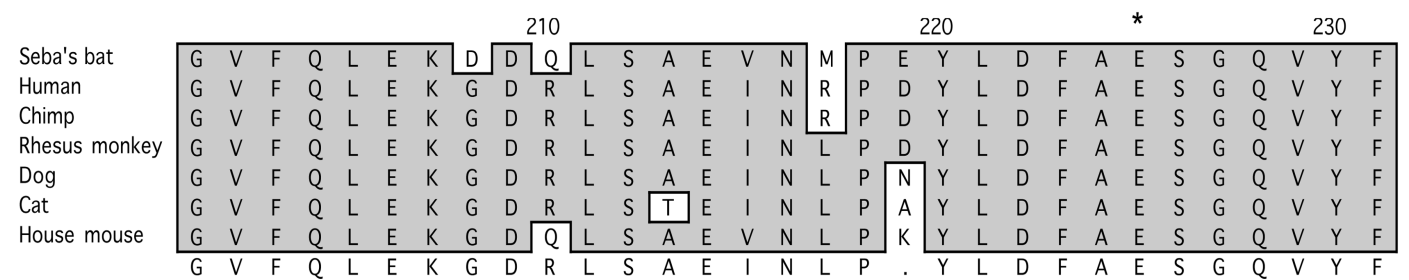

Fig. (1). Amino acid alignment of TNF sequences. Seba's short-tailed bat cDNA was translated using the default translation table in MacVector DNA analysis software. The human, chimp, rhesus monkey, dog, cat and mouse TNF sequences were imported into MacVector from NCBI (accession numbers in Table 2) and aligned using the default parameters in the clustalw algorithm. Regions of sequence outlined in dark gray represent either consensus amino acid identities or similarities and white areas indicate non-similar amino acids. The bar that is denoted TM represents the region of TNF that is involved in the transmembrane domain. Residues marked with asterisks represent conserved residues that are involved in biological function of TNF.

TNF [26], and are conserved in Seba's short-tailed bat polypeptide.

\section{IL-10}

The cloned cDNA fragment of Seba's short-tailed bat IL10 was $453 \mathrm{nt}$ in length and encodes approximately $80 \%$ of the IL-10 polypeptide (151 residues) (Fig. 2). The polypeptide has $78 \%$ identity and $84 \%$ similarity to the human IL-10 sequence [27], and 74\% identity and $82 \%$ similarity to Leschenault's Rousette bat IL-10 [15]. The amino acid residues for the first four helices are highly conserved in the Seba's short-tailed bat sequence (helix A, residues 42-65; B, 73-82; C, 84-106; D, 111-134) [28]. The sequence is incomplete for the fifth helix. The four 


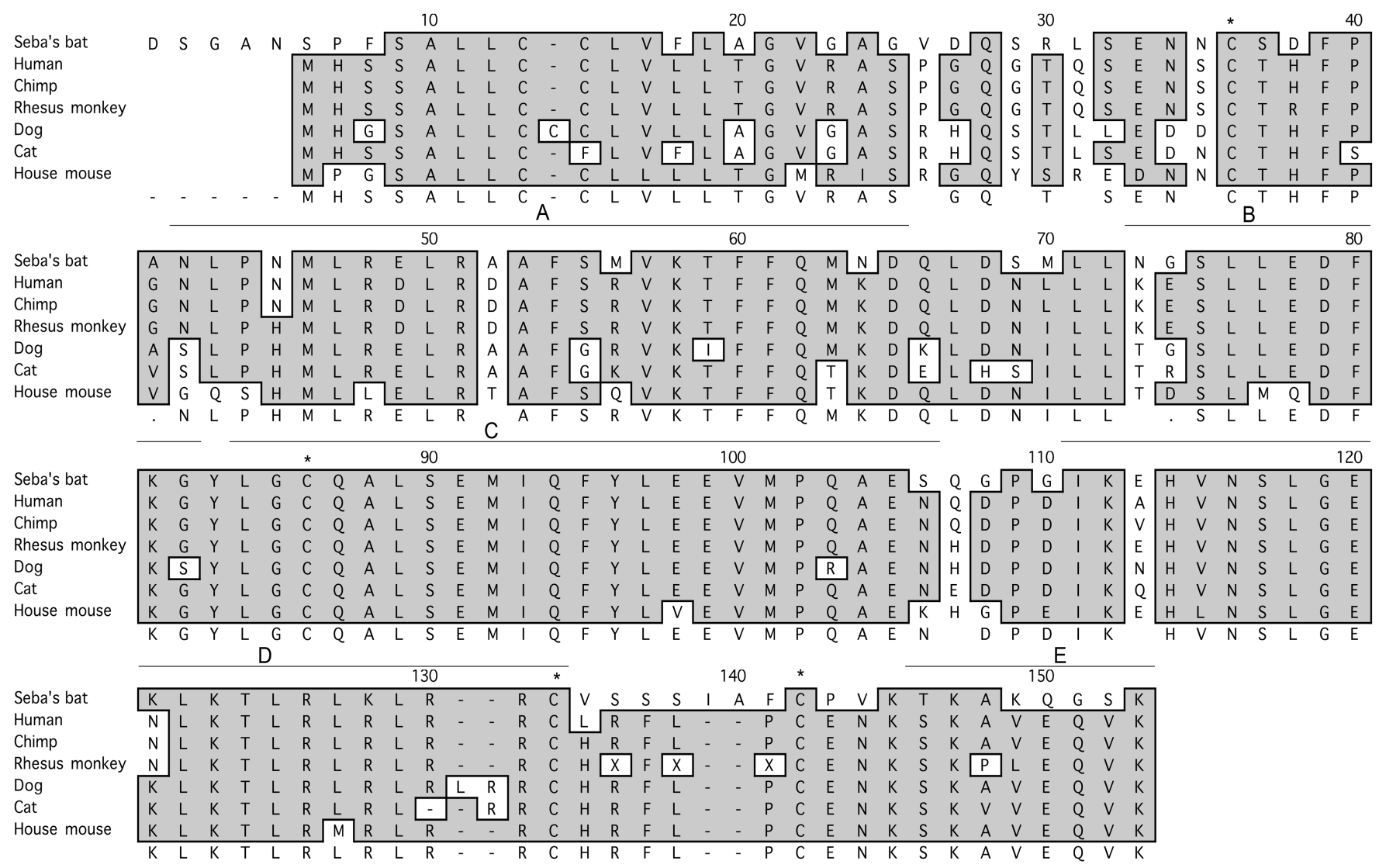

Fig. (2). Amino acid alignment of IL-10. Seba's short-tailed bat IL-10 amino acid sequence was aligned with other orthologous mammalian IL-10 sequences as described in Fig. (1). Lettered boxes above the sequences indicate helix regions and the four asterisks denote conserved cysteines that form intrachain disulfide bonds.

conserved cysteine residues that are responsible for interchain disulfide bonds [29] are also present in the bat sequence.

\section{IL-23a}

The cloned fragment of IL-23a was $594 \mathrm{nt}$ and encodes nearly $95 \%$ of IL-23a gene (Fig. 3). The nucleotide sequence encodes nearly all of the IL-10 polypeptide (197 residues) and exhibits approximately $74 \%$ identity and $81 \%$ similarity to human IL-23a. The bat sequence contains all four cysteine residues that are involved in intrachain disulfide bonding (residues 48, 84, 88, and 100) and also includes all four helices (helix A, residues 39-58; B, 98-121; C, 127-147; D, 166-196) [30].

\section{GM-CSF}

The nucleotide sequence for Seba's short-tailed bat GMCSF gene is $290 \mathrm{nt}$ and encodes the first 96 residues ( $69 \%)$ of the bat polypeptide. This region is highly conserved with a 25-residue signal peptide present (L, residues 1-25, Fig. 4) and shares $71 \%$ identity and $78 \%$ similarity to human GMCSF. The bat polypeptide contains a partially conserved helix A that is involved in binding to the GM-CSF receptor (A, residues 29-44) [21,31,32], which differs by 6 amino acids compared to the mouse, and by 8 amino acids compared to the human sequences. The $\beta 1$ strand that is involved in receptor binding is also present in this sequence (residues 59-61). Helix B is highly conserved (B, residues 72-84), and a partial helix $C$ is encoded in the polypeptide (C, residues 91-97) [32]. The bat sequence also encodes a conserved cysteine residue (residue 71) that is used for the intrachain disulfide bond [31]. The paired cysteine is located near the C-terminus of the polypeptide in other species and would be found in the uncloned region of the bat sequence presented here.

\section{Phylogenetic Analysis}

The alignment of TNF sequences included a total of 246 characters, of which 190 were variable and 45 were parsimony informative. The alignment of IL-10 sequences included a total of 188 characters, of which 128 were variable and 51 were parsimony informative. The alignment of IL-23a sequences included a total of 235 characters, of which 174 were variable and 39 were parsimony informative. The alignment of GM-CSF sequences included a total of 156 characters, of which 124 were variable and 56 were parsimony informative.

Analysis of the TNF data resulted in 5 equally parsimonious trees (C.I. $=0.9054$; R.I. $=0.5272$; length $=$ 349). The consensus tree placed Seba's short-tailed bat as unresolved at the base of the tree with house mouse, horse, and chicken (Fig. 5A). The bootstrap analysis strongly 


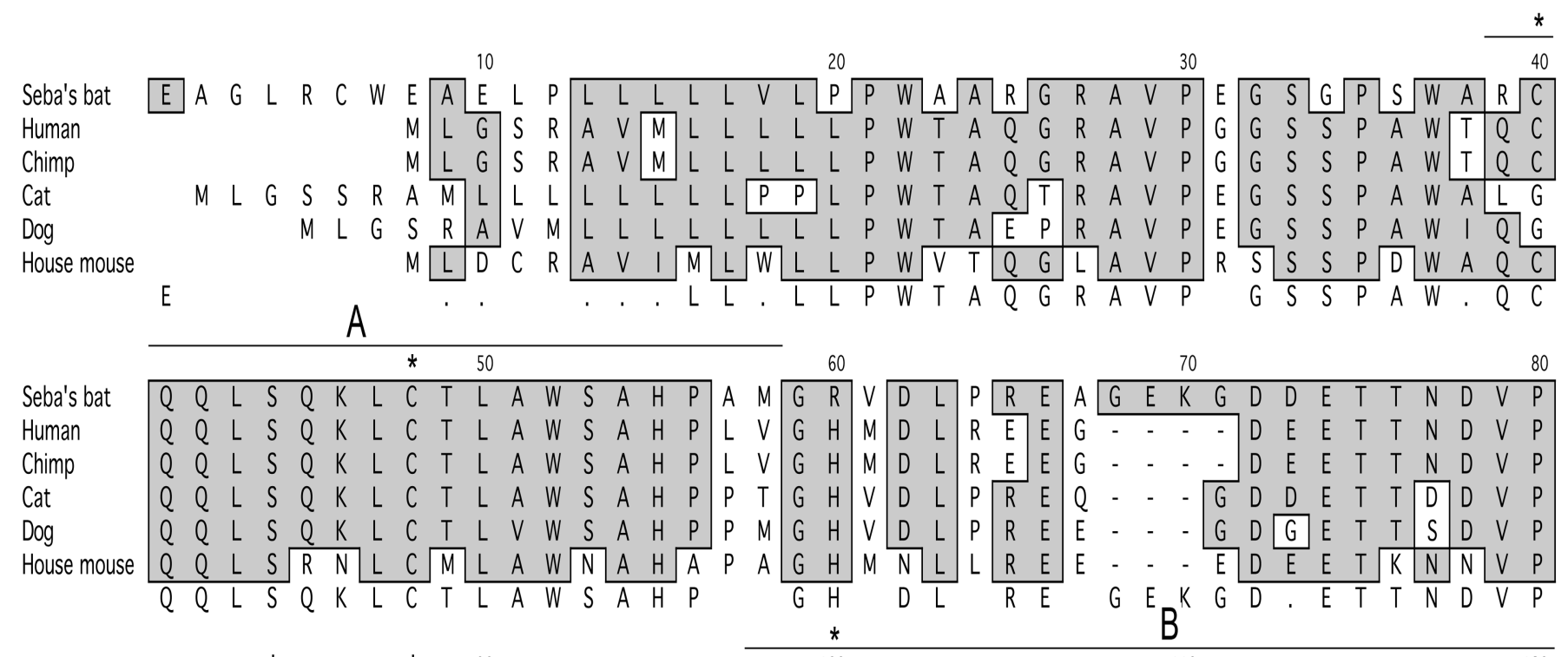

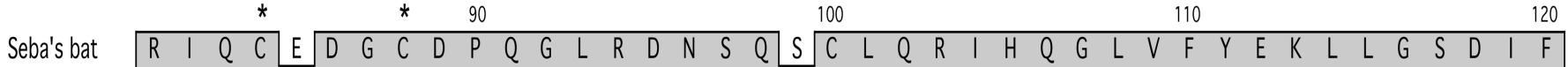

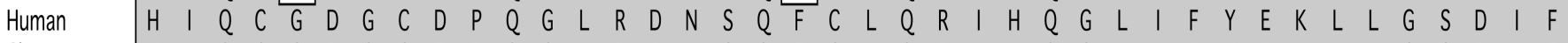

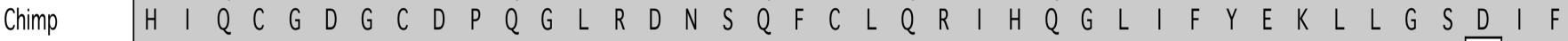

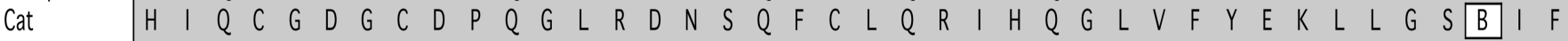

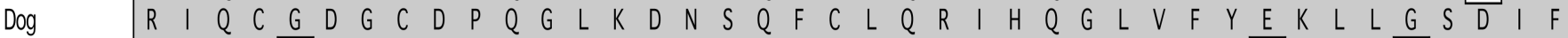

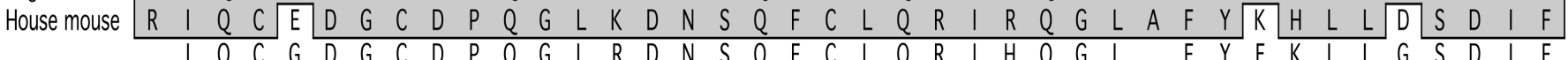
C

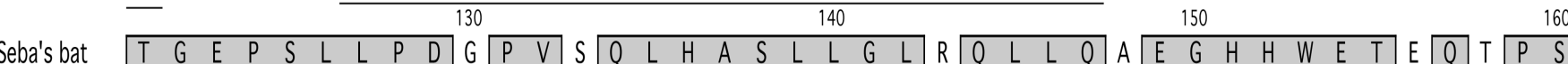

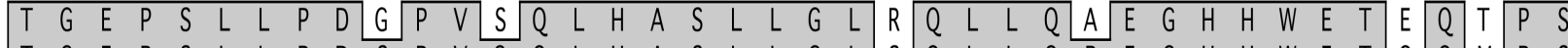

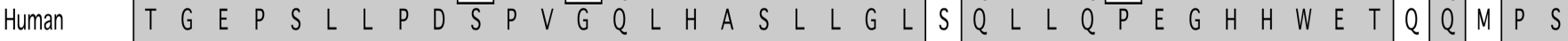

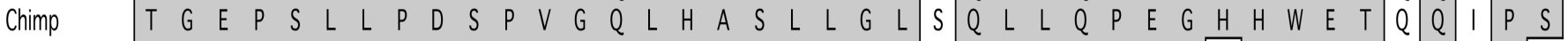

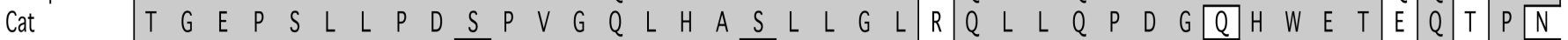

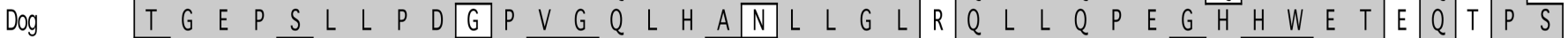

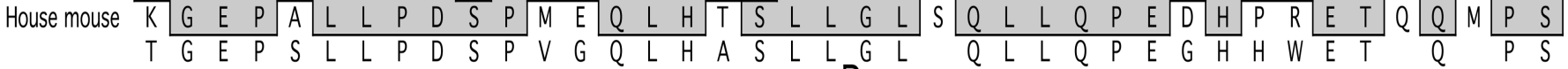
D

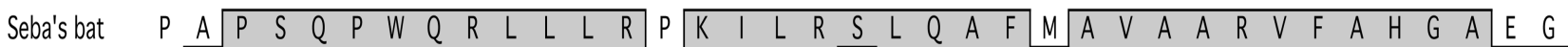

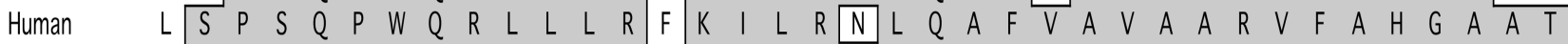

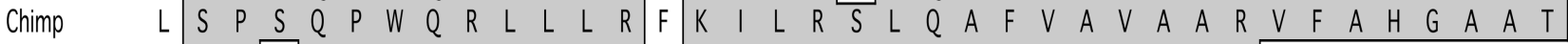
\begin{tabular}{ll|ll|lllllllllllllllllllllllllll} 
Cat & $P$ & $S$ & $P$ & $N$ & $Q$ & $P$ & $W$ & $Q$ & $R$ & $L$ & $L$ & $L$ & $R$ & $F$ & $K$ & $I$ & $L$ & $R$ & $S$ & $L$ & $Q$ & $A$ & $F$ & $V$ & $A$ & $V$ & $A$ & $A$ & $R$
\end{tabular} \begin{tabular}{ll|llllllllllllllllllllllllllllllllllll} 
Dog & $P$ & $S$ & $P$ & $S$ & $Q$ & $P$ & $W$ & $Q$ & $R$ & $L$ & $L$ & $L$ & $R$ & $L$ & $K$ & $I$ & $L$ & $R$ & $S$ & $L$ & $Q$ & $A$ & $F$ & $V$ & $A$ & $V$ & $A$ & $A$ & $R$ & $V$ & $F$ & $A$ & $H$ & $G$ & $A$ & $A$ & $T$ \\
\hline
\end{tabular}

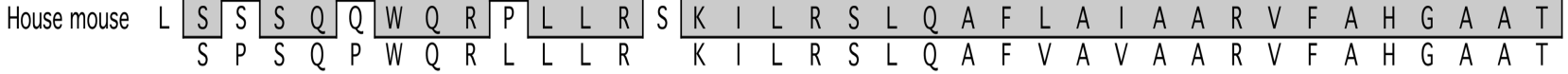

Fig. (3). Amino acid alignment of IL-23a. Seba's short-tailed bat IL-23a amino acid sequence was aligned with other IL-23a sequences as described in Fig. (1). Lettered boxes above the sequences denote four helix regions and the five asterisks indicate conserved cysteine residues.

supported a clade with human, chimpanzee, and rhesus monkey (99\% bootstrap), with human and chimpanzee exhibiting a sister relationship within that clade (71\% bootstrap).

Analysis of the IL-10 data resulted in 2 equally parsimonious trees $($ C.I. $=0.8732$; R.I. $=0.6500$; length $=$ 276). The consensus tree placed Seba's short-tailed bat as basal to a clade containing domestic dog and domestic cat (Fig. 5B). However, bootstrap analysis did not support the placement of Seba's short-tailed bat. The only clade that was strongly supported contains human, chimpanzee, and rhesus monkey ( $96 \%$ bootstrap), with human and chimpanzee exhibiting a sister relationship within that clade ( $96 \%$ bootstrap). 


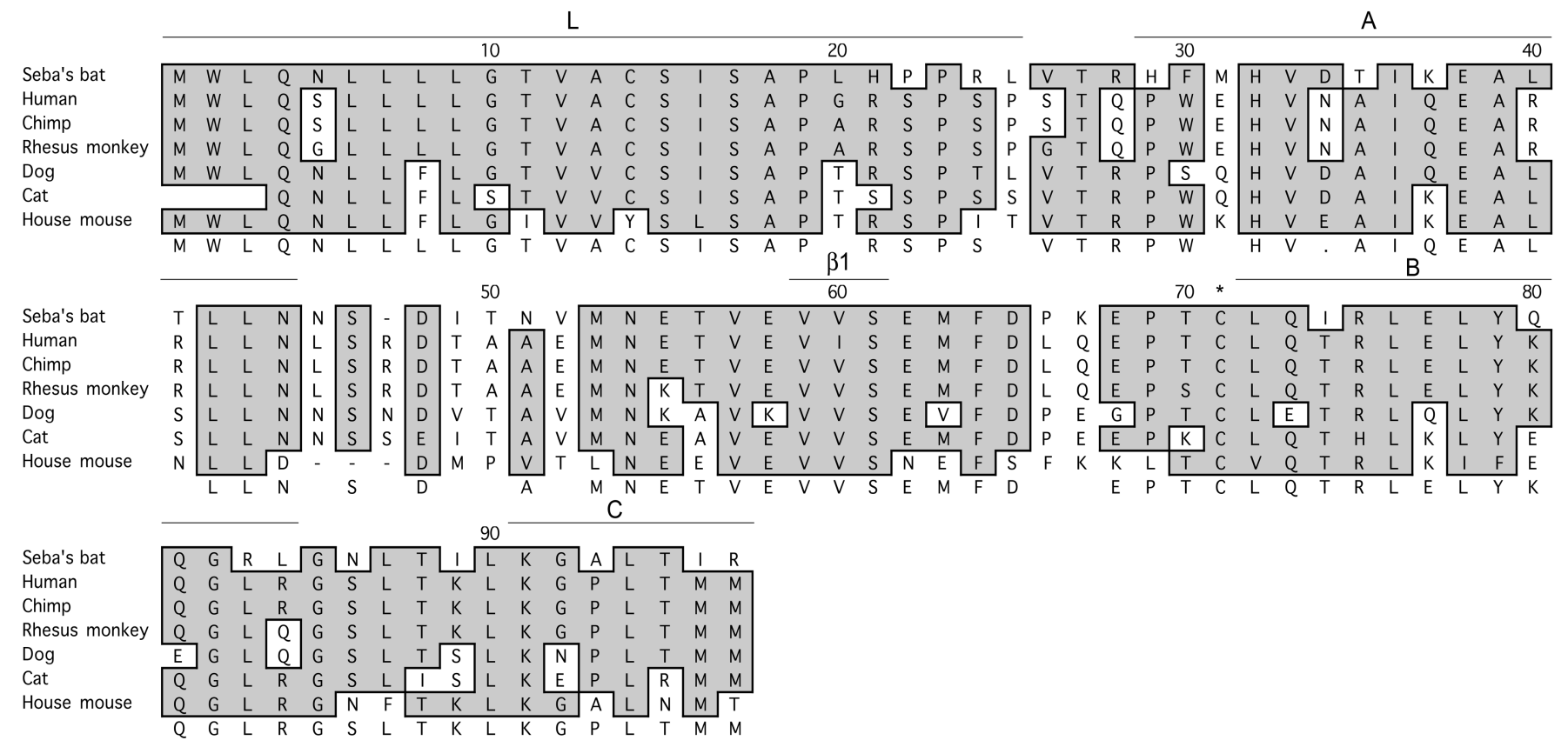

Fig. (4). Amino acid alignment of GM-CSF. Seba's short-tailed bat GM-CSF amino acid sequence was aligned with other mammalian GMCSF sequences as described in Fig. (1). The region denoted with an $\mathrm{L}$ indicates a 25 amino acid signal peptide and the $\beta 1$ is the domain involved in receptor binding. Conserved helices A, B and C are denoted by bars above the sequences.

Analysis of the IL-23a data resulted in single most parsimonious tree $($ C.I. $=0.9313$; R.I. $=0.6901$; length $=$ 320). Within the tree Seba's bat was placed basal to a clade containing all of the placental mammals except house mouse (Fig. 5C). Bootstrap analysis strongly supported the clade grouping Seba's bat with all of the placental mammals except house mouse (92\% bootstrap) and the clade containing human, chimpanzee, and rhesus monkey $(100 \%$ bootstrap), with human and chimpanzee exhibiting a sister relationship within that clade (91\% bootstrap).

Analysis of the GM-CSF data resulted in 2 equally parsimonious trees $($ C.I. $=0.8859$; R.I. $=0.6264$; length $=$ 298). The consensus tree placed Seba's short-tailed bat as unresolved relative to all of the mammals except for mouse, which was unresolved at the base of the tree with chicken (Fig. 5D). The bootstrap analysis strongly supported a clade with human, chimpanzee, and rhesus monkey $(100 \%$ bootstrap), with human and chimpanzee exhibiting a sister relationship within that clade ( $71 \%$ bootstrap).

\section{DISCUSSION}

Until recently, bats have been underappreciated as reservoir hosts for pathogenic viruses. The relationships between bats and their viruses are poorly understood. Many zoonotic viruses hosted by other mammals may establish persistent infections without significant pathological consequences. Identification of the underlying mechanisms governing non-pathogenic infections of reservoirs may provide clues to virus ecology as well as the pathogenesis in human diseases caused by these viruses [1,33].

We have begun to develop laboratory models to understand the immunological relationships between viruses and their bat hosts. We have successfully cloned and sequenced several cytokine genes from bats and have shown that these sequences are substantially conserved among known mammalian orthologs.

TNF is thought to play a prominent role in pathogenesis of many infectious diseases, including hantavirus cardiopulmonary syndrome, dengue hemorrhagic fever and dengue shock syndrome [34]. It is a chemotactic inflammatory cytokine secreted by various immune cells and is expressed as a membrane-bound homotrimer that is cleaved by a cellular protease imbedded in the plasma membrane; its release induces inflammation by causing vascular leakage [35]. The sequence of TNF from Seba's short-tailed bat is highly similar to TNF of other species and, other than a three amino acid deletion at positions 64-66, contains no unusual differences from those sequences. Interestingly, Seba's fruit bat TNF has fewer identities, but more similarities to human TNF than it does to Leschenault's Rousette bat TNF.

IL-10 is a noncovalently-linked homodimer secreted by many cells, including some regulatory $\mathrm{T}$ cell subsets, and is capable of suppressing inflammatory responses [36, 37]. Each monomer is composed of six alpha helices and four conserved cysteine residues that are responsible for intrachain disulfide bond formation [28]. The bat polypeptide also is highly similar to IL-10 from vertebrates of other species, especially within their functional domains. The Seba fruit bat sequence has more similarity and identity to human IL-10 than it does to Leschenault's Rousette bat IL-10.

IL-23a is in the interleukin- 12 family and is coexpressed with IL-12p40 to form a disulfide-linked heterodimer [30]. It 

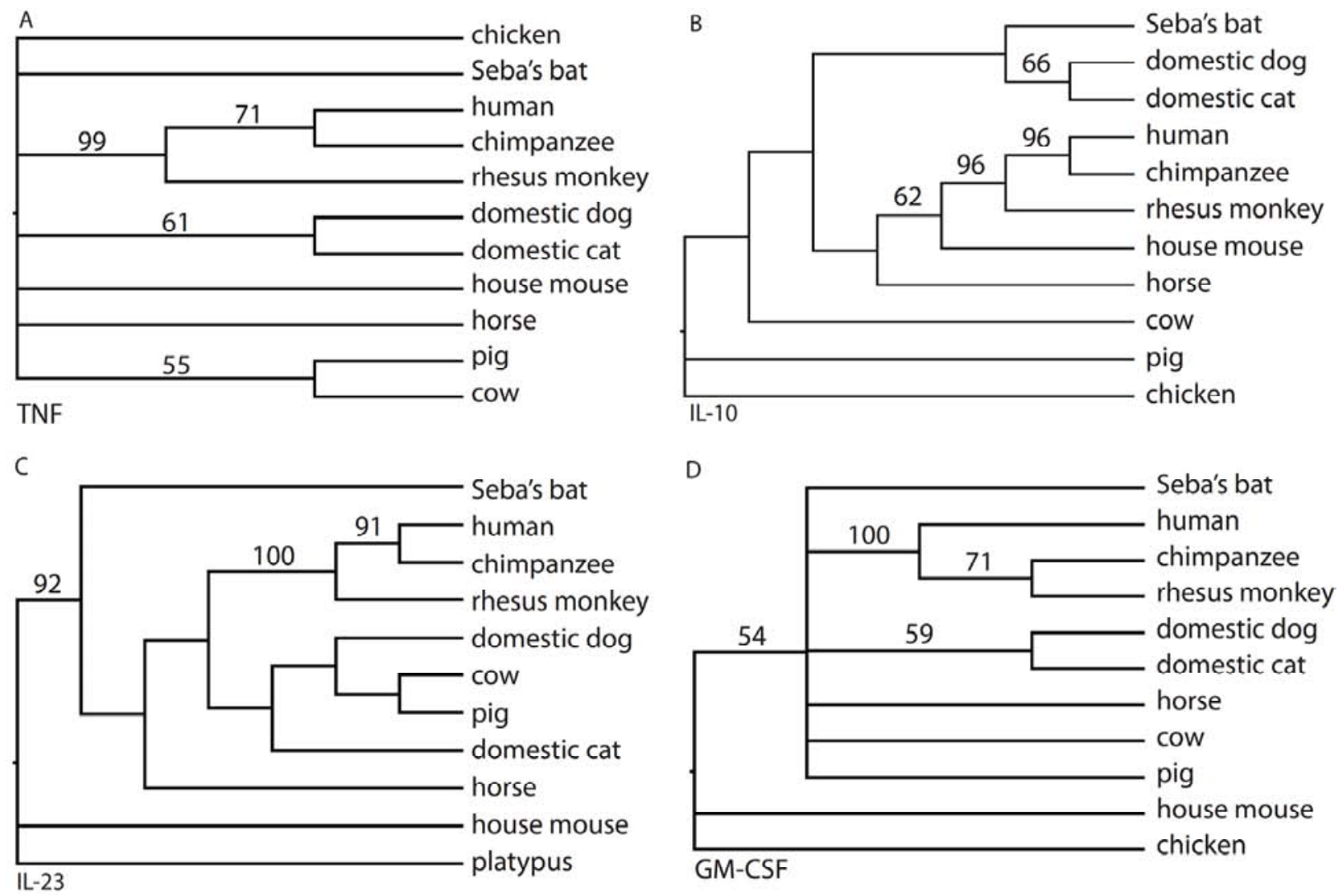

Fig. (5). Most parsimonious phylogenetic tree reconstructions based on cytokine gene amino acid sequences. Amino acid sequences (Table 3) were analyzed with PAUP* to reconstruct trees. Genes are as follows: A, TNF; B, IL-10; C, IL-23a; D, GM-CSF. Bootstrap values greater than $50 \%$ are shown above the branches.

is secreted from human and mouse dendritic cells generated in the presence of GM-CSF and IL-4, can induce IFN $\gamma$ responses and promote proliferation of memory $\mathrm{T}$ cells in mice [38]. It is important for initiation of the adaptive immune response to some infections. The bat polypeptide is highly similar within domains to IL-23a of other mammalian species; however, it possesses a three amino acid insertion (GEK) at positions 68-70, between helices $A$ and $B$, a region with unrecognized biological significance.

GM-CSF is a glycoprotein that can stimulate the growth and differentiation of many immune cells, including bone marrow stem cells [39, 40]. It is secreted by a variety of cells, including Th1 cells, controls the activities of a variety of phagocytic cells, is an important stimulator for the growth and development of immune cells, and is routinely used for in vitro propagation of dendritic cells from bone marrow progenitors [41]. Although the sequence of Seba's shorttailed bat GM-CSF is highly similar to orthologous sequences, it has several differences in helix A, a receptor binding domain.

Phylogenetic reconstructions did not provide clear resolution of the relationship between Seba's short-tailed bat and other placental mammals. Phylogenetic trees from three cytokine genes (TNF, IL-23a, and GM-CSF) placed Seba's short-tailed bat in an unresolved position at or near the base of the tree. IL-10 provided the best phylogenetic resolution among taxa, placing Seba's short-tailed bat in a clade containing domestic dog and domestic cat. This placement corresponds to recent mammalian phylogenetic analyses, which group bats, dogs, and cats into the Superorder Laurasiatheria [42]. However, Superorder Laurasiatheria also contains cattle, horse, and pig, which are dispersed throughout the IL-10 phylogenetic tree. The general lack of phylogenetic resolution indicates that additional taxonomic sampling will be required to accurately document the evolutionary divergence of these cytokine genes.

We are continuing to clone and sequence additional cytokine genes and other immune related transcription factors of Seba's short-tailed bat to assess immune responses. We believe these primer sets could be used for amplifying and cloning orthologous sequences from bats of many other species. In addition, the little brown bat (Myotis lucifugus) genome has recently being sequenced to deep coverage ( $7 x$, Broad Institute, Cambridge, MA), which should provide an important genomic resource for research efforts regarding reservoir bat host responses to viral infections.

\section{ACKNOWLEDGEMENTS}

This project was supported by the University of Northern Colorado Research Enhancement and Development Initiative, NIH grant AI089419 and NIH Emerging Virus Diseases Unit contract AI25489. We thank Drs. Katherine V. Holmes, Department of Microbiology, University of Colorado Health Sciences Center, Aurora, Colorado, Robert B. Tesh, Department of Pathology, Center for Tropical Diseases, University of Texas Medical Branch, Galveston, Texas for advice and diagnostic testing of colony bats, and 
Sara Shields, UNC, for assistance in preparing alignment figures.

\section{CONFLICTS OF INTEREST}

None declared.

\section{REFERENCES}

[1] Calisher CH, Childs JE, Field HE, Holmes KV, Schountz T. Bats: important reservoir hosts of emerging viruses. Clin Microbiol Rev 2006; 19: 531-45.

[2] Halpin K, Young PL, Field HE, Mackenzie JS. Isolation of Hendra virus from pteropid bats: a natural reservoir of Hendra virus. J Gen Virol 2000; 81: 1927-32.

[3] Selvey L, Wells ARM, McCormack JG, et al. Infection of humans and horses by a newly described morbillivirus. Med J Aust 1995; 162: 6425 .

[4] Chua KB, Koh CL, Hooi PS, et al. Isolation of Nipah virus from Malaysian Island flying-foxes. Microbes Infect 2002; 4: 145-51.

[5] Reynes JM, Counor D, Ong S, et al. Nipah virus in Lyle's flying foxes, Cambodia. Emerg Infect Dis 2005 11: 1042-7.

[6] Rupprecht CE, Smith JS, Fekadu M, Childs JE. The ascension of wildlife rabies: a cause for public health concern or intervention?. Emerg Infect Dis 1995; 1: 107-14.

[7] Lau SK, Woo PC, Li KS, et al. Severe acute respiratory syndrome coronavirus-like virus in Chinese horseshoe bats. Proc Natl Acad Sci USA 2005; 102: 14040-5.

[8] Leroy E, Kumulungui MB, Pourrut X, et al. 2005. Fruit bats as reservoirs of Ebola virus. Nature 438: 575-6.

[9] Wong S, Lau S, Woo P, Yuen KY. Bats as a continuing source of emerging infections in humans. Rev Med Virol 2007; 17: 67-91.

[10] Baker M.L, Tachedjian M, Wang LF. Immunoglobulin heavy chain diversity in Pteropid bats: evidence for a diverse and highly specific antigen binding repertoire. Immunogenetics 2010; 62: 173-84.

[11] Butler JE, Wertz N, Zhao Y, et al. The two suborders of chiropterans have the canonical heavy-chain immunoglobulin [Ig] gene repertoire of eutherian mammals. Dev Comp Immunol 2010; 35: 273-84.

[12] Cowled C, Baker M, Tachedjian M, Zhou P, Bulach D, Wang LF. Molecular characterisation of Toll-like receptors in the black flying fox Pteropus alecto. Dev Comp Immunol 2010; 35: 7-18.

[13] Fujii HS, Watanabe D, Yamane N, et al. Functional analysis of Rousettus aegyptiacus "signal transducer and activator of transcription 1" (STAT1). Dev Comp Immunol 2010; 34: 598-602.

[14] Kepler TB, Sample C, Hudak J, et al. Chiropteran types I and II interferon genes inferred from genome sequencing traces by a statistical gene-family assembler. BMC Genomics 2010; 11: 444.

[15] Iha K, Omatsu T, Watanabe S, et al. Molecular cloning and expression analysis of bat toll-like receptors 3, 7 and 9. J Vet Med Sci 2009 72: 217-20.

[16] Baillie GJ, van de Lagemaat LN, Baust C, Mager DL. Multiple groups of endogenous betaretroviruses in mice, rats, and other mammals. J Virol 2004; 78: 5784-98.

[17] Calisher CH, Kinney RM, Lopes OD, et al. Identification of a new Venezuelan equine encephalitis virus from Brazil. Am J Trop Med Hyg 1982; 31: 1260-72.

[18] Carrington CV, Foster JE, Zhu HC, et al. Detection and phylogenetic analysis of group 1 coronaviruses in South American bats. Emerg Infect Dis 2008; 14: 1890-3.

[19] Scherer WF, Dickerman RW, Campillo-Sainz C, Zarate ML, Gonzales E. Ecologic studies of Venezuelan encephalitis virus in southeastern Mexico. V. Infection of domestic animals other than equines. Am J Trop Med Hyg 1971; 20: 989-93.

[20] Wilgenbusch JC, Swofford D. Inferring evolutionary trees with PAUP*. Curr Protoc Bioinformatics 2003; Chapter 6: Unit 6.4.

[21] Schountz T, Green B, Davenport A, et al. Cloning and characterization of deer mouse (Peromyscus maniculatus) cytokine and chemokine cDNAs. BMC Immunol 2006; 5: 1.
[22] Herbst MM, Prescott J, Palmer AD, Schountz T. Sequence and expression analysis of deer mouse interferon-gamma, interleukin-10, tumor necrosis factor, and lymphotoxin-alpha. Cytokine 2002; 17: 20313.

[23] Pennica D, Nedwin GE, Hayflick JS, et al. Human tumour necrosis factor: precursor structure, expression and homology to lymphotoxin. Nature 1984; 312: 724-9.

[24] Narachi MA, Davis JM, Hsu YR, Arakawa T. Role of single disulfide in recombinant human tumor necrosis factor-alpha. J Biol Chem 1987; 262: 13107-10.

[25] Yamamoto R, Wang A, Vitt CR, Lin LS. Histidine-15: an important role in the cytotoxic activity of human tumor necrosis factor. Protein Eng 1989; 2: 553-8.

[26] Van Ostade X, Tavernier J, Prange T, Fiers W. Localization of the active site of human tumour necrosis factor (hTNF) by mutational analysis. Embo J 1991; 10: 827-36.

[27] Vieira P, De Waal-Malefyt R, Dang MN, et al. Isolation and expression of human cytokine synthesis inhibitory factor cDNA clones: homology to Epstein-Barr virus open reading frame BCRFI. Proc Natl Acad Sci USA 1991; 88: 1172-6.

[28] Zdanov A, Schalk-Hihi C, Gustchina A, Tsang M, Weatherbee J, Wlodawer A. Crystal structure of interleukin-10 reveals the functional dimer with an unexpected topological similarity to interferon gamma. Structure 1995; 3: 591-601.

[29] Windsor WT, Syto R, Tsarbopoulos A, et al. Disulfide bond assignments and secondary structure analysis of human and murine interleukin 10. Biochemistry 1993; 32: 8807-15.

[30] Oppmann B, Lesley B, Blom JC, et al. Novel p19 protein engages IL12 p40 to form a cytokine, IL-23, with biological activities similar as well as distinct from IL-12. Immunity 2000; 13: 715-25.

[31] Diederichs K, Boone T, Karplus PA. Novel fold and putative receptor binding site of granulocyte-macrophage colony-stimulating factor. Science 1991; 254: 1779-82.

[32] Walter MR, Cook WJ, Ealick SE, Nagabhushan TL, Trotta PP, Bugg CE. Three-dimensional structure of recombinant human granulocytemacrophage colony-stimulating factor. J Mol Biol 1992; 224: 1075-85.

[33] Alcami A, Koszinowski UH. Viral mechanisms of immune evasion. Trends Microbiol 2000; 8: 410-8.

[34] Kurane I, Rothman AL, Livingston PG, et al. Immunopatho-logic mechanisms of dengue hemorrhagic fever and dengue shock syndrome Arch Virol Suppl 1994; 9: 59-64.

[35] Banner DW, D'Arcy A, Janes W, et al. Crystal structure of the soluble human $55 \mathrm{kd}$ TNF receptor-human TNF beta complex: implications for TNF receptor activation. Cell 1993; 73: 431-45.

[36] Izcue A, Coombes JL, Powrie F. Regulatory T cells suppress systemic and mucosal immune activation to control intestinal inflammation. Immunol Rev 2006; 212: 256-71.

[37] Rouse BT, Sarangi PP, Suvas S. Regulatory T cells in virus infections Immunol Rev 2006; 212: 272-86.

[38] Cua DJ, Sherlock J, Chen Y, et al. Interleukin-23 rather than interleukin-12 is the critical cytokine for autoimmune inflammation of the brain. Nature 1993; 421: 744-8.

[39] Chen-Woan M, Delaney CP, Fournier V, et al. A new protocol for the propagation of dendritic cells from rat bone marrow using recombinant GM-CSF, and their quantification using the mAb OX-62. J Immunol Methods 1995; 178: 157-71.

[40] Scheicher C, Mehlig M, Zecher R, Reske R. Dendritic cells from mouse bone marrow: in vitro differentiation using low doses of recombinant granulocyte-macrophage colony-stimulating factor. J Immunol Methods 1992; 154: 253-64.

[41] Lutz MB, Kukutsch N, Ogilvie AL, et al. An advanced culture method for generating large quantities of highly pure dendritic cells from mouse bone marrow. J Immunol Methods 1999; 223: 77-92.

[42] Prasad AB, Allard MW, Green ED. Confirming the phylogeny of mammals by use of large comparative sequence data sets. Mol Biol Evol 2008; 25: 1795-808. 